\title{
5 Research Square

\section{Comparison of the Jcerity Endoscoper Airway with the LMA Supreme for Airway Management in Patients Undergoing Cerebral Aneurysm Embolization}

\section{Junfei Zhou}

First Affiliated Hospital of Zhengzhou University

Lu Li

First Affiliated Hospital of Zhengzhou University

\section{Fang Wang}

Second Affiliated Hospital of Zhengzhou University

Yunqi Lv ( $D$ lyq260703@gmail.com )

First Affiliated Hospital of Zhengzhou University

\section{Research Article}

Keywords: laryngeal mask airway, airway management, cerebral aneurysm embolization, anesthesiology

Posted Date: August 27th, 2021

DOI: https://doi.org/10.21203/rs.3.rs-847303/v1

License: (c) (1) This work is licensed under a Creative Commons Attribution 4.0 International License.

Read Full License 


\section{Abstract \\ Background}

Interventional embolization of cerebral aneurysms often requires anticoagulation and antiplatelet therapy during perioperative period. A new type of laryngeal mask airway (Jcerity Endoscoper Airway)with a unique design may cause less oropharyngeal injury and bleeding for patients receiving perioperative anticoagulation. This study sought to compare the efficacy, safety and complications of Jcerity Endoscoper airwayvs LMA((Laryngeal Mask Airway) Supreme in the procedure of cerebral aneurysm embolization.

\section{Methods}

In this prospective, randomised clinical trial, 182 adult patients with American Society of Anesthesiologists class I-II scheduled for interventional embolization of cerebral aneurysms were randomly allocated into the Jcerity Endoscoper airway group and the LMA Supreme group. We compared success rate of LMA implantation, ventilation quality, airway sealing pressure, peak airway pressure, degree of blood staining, postoperative oral hemorrhage, sore throat and other complications between the groups.

\section{Results}

There were no significant differences between the groups in terms of success rate of LMA implantation, ventilation quality, airway sealing pressure or airway peak pressure. The LMA Supreme group showed a significantly higher degree of blood staining than the Jcerity Endoscoper airway group when the laryngeal mask airway was removed $(P=0.04)$, and there were also more oral hemorrhages and pharyngeal pain than in the the Jcerity Endoscoper airway group $(P=0.03, P=0.02)$. No differences were observed between groups in terms of other complications related to the LMA.

\section{Conclusions}

The Jcerity Endoscoper airway can be safely and effectively used for airway management in patients undergoing cerebral aneurysm embolization, which can significantly reduce airway complications related to perioperative anticoagulation.

\section{Introduction}

With the popularization of endovascular techniques, interventional embolization of unruptured cerebral aneurysms with general anesthesia under digital subtraction angiography (DSA) has become the primary 
treatment [1-6]. In order to avoid aneurysm rupture and bleeding, the maintenance of hemodynamic stability during the perioperative period is important factor that should be taken into account $[7,8]$.

During cerebral aneurysm embolization, there is less stimulation because it is generally performed through femoral artery puncture and catheter placement. To ensure that the patient remains immobile and fully sedated during the operation, excessive depth of anesthesia and excessive analgesia are not required, so as to ensure that the patient can wake up immediately after the operation, it is convenient to evaluate the patient's neurological function status [7, 9]. Patients who intend to undergo cerebral aneurysm embolization generally need anticoagulation and antiplatelet therapy before surgery $[10,11]$, and anticoagulation treatment during and after surgery. This anticoagulation treatment would become a challenge for the airway management during the anesthesia.

Traditionally, Intubation rarely causes the oropharyngeal bleeding. However, conducting tracheal intubation often requires a deeper depth of anesthesia. Moreover, extubation may provoke coughing and have aneurysm rupture risk during the process of recovery from anesthesia[12].Combined with the characteristics of cerebral aneurysm embolization, we often use LMA Supreme during general anesthesia for cerebral aneurysm embolization, which contributes to the stability of hemodynamics and rapid recovery of patients during the perioperative period. However, it often causes or opharyngeal injury in patients receiving anticoagulant and antiplatelet therapy due to the design of dual lumen laryngeal mask. The Jcerity Endoscoper Airway (Zhejiang Jcerity Medical Technology Co., Ltd, Huzhou, China) was originally designed for painless gastroscopy and treatment (Fig. 1). Unexpectedly, the special material and bionic structure may give the device unique advantages forcerebral aneurysm embolization. Therefore, the main purpose of this study was to compare the efficacy, safety and complications associated with Jcerity Endoscoper Airway and LMA Supreme in aneurysm embolization, so as to further optimize the anesthesia airway management mode of such operations.

\section{Materials And Methods}

We conducted a randomized, controlled trial between April 2021 and July 2021 in the First Affiliated Hospital of Zhengzhou University in China registered at Chinese Clinical Trial Registry (ChiCTR2100044133).This trial was approved by the ethics committee of the First Affiliated Hospital of Zhengzhou University(2019-KY-276), and each patient provided informed written consent. We studied 182 adult patients with physical status of I-II according to the American Society of Anesthesiologists, who were scheduled for elective cerebral aneurysm embolization under general anesthesia. Patients were divided in to an Jcerity Endoscoper Airway group and an LMA Supreme group. Exclusion criteria were as follows: neck deformity; high risk of aspirating stomach contents; known or expected difficult airways; obesity (body mass index [BMI] > $30 \mathrm{~kg} \cdot \mathrm{m}^{-2}$; estimated operation time > 3 hours; reduced lung compliance; incisor defect; mouth opening limitations. The LMA size was selected according to the patient's weight. All LMA insertions were performed by a senior anesthesiologist who had more than 100 successful LMA insertion experiencesn (Fig. 2 for the study flow diagram). 
Thirty minutes before anesthesia, all patients were given penehyclidine hydrochloride $0.01 \mathrm{mg} \cdot \mathrm{kg}$ by intravenous injection. All patients were routinely monitored using electrocardiogram, pulse oximetry $\left(\mathrm{SpO}_{2}\right)$, non-invasive blood pressure measurement, and determination of end-tidal carbon dioxide and bispectral index (BIS). Before induction, patients were pre-oxygenated for no less than 3 minutes. Inducing drugs included etomidate $\left(0.2 \mathrm{To} 0.3 \mathrm{mg} \cdot \mathrm{kg}^{-1}\right)$, sufentanil $\left(0.1 \mathrm{ug} \cdot \mathrm{kg}^{-1}\right)$, cisatracurium $(0.15$ to $0.2 \mathrm{mg} \cdot \mathrm{kg}^{-1}$ ) and dezocine $0.1 \mathrm{mg} \cdot \mathrm{kg}^{-1}$. The selected Jcerity Endoscoper Airway or LMA Supreme was inserted according to the manufacturer's instruction manual. Before insertion, each LMA was lubricated with Obucaine gel. Volume controlled ventilation (tidal volume $6-8 \mathrm{ml} \cdot \mathrm{kg}^{-1}$ ) was used to verify the size and position of the LMA device. If the lungs could not be ventilated, another insertion attempt was made, during which time the jaw was allowed to be lifted. If the patient still couldn't be ventilated, the device changed to another size. If there was still no ventilation, the LMA was abandoned and replaced with endotracheal intubation. After the LMA was placed, agastric tube was inserted 40 to $55 \mathrm{~cm}$ to aspirate gastric contents. If the gastric tube couldn't be inserted, the patient was excluded from the study. Once ventilation was successful and the gastric tube was easily inserted, the LMA was secured with tape. Mechanical ventilation was performed at tidal volumes of $6-8 \mathrm{ml} \cdot \mathrm{kg}^{-1}$ and a certain frequency, and the end-expiratory $\mathrm{CO}_{2}$ was maintained in the normal range. End-expiratory carbon dioxide and pressure volume curves were recorded throughout the procedure.

During the operation, continuous infusion of sevoflurane and remifentanil was used to maintain the BIS between 40 and 60 . According to the length of the operation, cisatracurium was added to prevent head movement that was caused by respiratory confrontation due to the recovery of spontaneous breathing (head micromotion often produced artifacts under DSA and affected the operation). The number of LMA attempts with or without assistance was recorded by an independent observer, and the success rate of the first or second placement was recorded. We recorded the duration of implantation time (from the time of LMA insertion to the time of fixing LMA with tape). LMA ventilation quality was scored on a 3-point scale: (1) optimal ventilation was defined as normal thoracic expansion, normal pressure-volume curve and square wave diagram without air leakage; (2) ventilation difficulty was defined as peak airway pressure $>25 \mathrm{~cm} \mathrm{H}_{2} \mathrm{O}$ or severe air leakage related to mechanical ventilation failure; and (3) after all attempts to ventilate failed, the case was changed to endotracheal intubation. If ventilation difficulty was managed by adjusting the insertion depth, changing the head and neck position or adjusting the cuff volume, the score was recorded as 2 points. The peak inspiratory pressure was recorded at the beginning of controlled ventilation. Airway sealing pressure was measured by closing the expiratory valve and observing the balanced airway pressure under the fresh air flow of $3 \mathrm{~L} \cdot \mathrm{min}^{-1}$. When the peak airway pressure did not rise or there was air leakage in the mouth, the airway pressure was the maximum leakage pressure. If the maximum air leakage pressure was less than $15 \mathrm{~cm} \mathrm{H}_{2} \mathrm{O}$, the LMA was replaced. If there was gas leakage in the mouth, we opened the expiratory valve to avoid alveolar trauma. If the maximum pressure reached $40 \mathrm{~cm} \mathrm{H}_{2} \mathrm{O}$, we recorded $40 \mathrm{~cm} \mathrm{H}_{2} \mathrm{O}$ as the airway sealing pressure. Airway sealing pressure was recorded at various time points (T1: immediately after LMA implantation; $\mathrm{T} 2$ : $15 \mathrm{~min}$ after T1; T3: 30min after T1; and T4: at the end of the operation). After recording the airway sealing 
pressure immediately after the LMA implantation, a 4.0-mm fiber optic bronchoscope (FBS) (F1-10RBS, Pentax, Japan) was introduced near the end of LMA by the same anesthesiologist familiar with fiberoptic bronchoscopy. If resistance was encountered, operated the tip of fiberoptic bronchoscope and classified the laryngoscope view. The optimal position of LMA was defined using fiberoptic bronchoscopy. If the tip of the laryngeal mask was located behind the arytenoid cartilage, it could be seen that the epiglottis had not been folded or blocked the airway, and the vocal cords could be seen. Any deviation from these standards was considered sub-optimal positioning.

The degree of blood staining was recorded after the laryngeal mask was removed. The criteria for judging the degree of blood staining of LMA were as follows: if a small amount of blood was detected on the pulled LMA, it was recorded as "mild"; if the blood was limited to a quarter of the LMA surface, it was classified as "moderate"; if the blood covers at least half of the LMA surface, it is classified as "severe". In case of moderate or above blood staining, the oral mucosal damage was observed using laryngoscopy 6 hours after operation).

We recorded complications related to airway management: tooth or mucosal trauma; cough; hiccup; laryngospasm; bronchospasm; high inspiratory pressure $\left(>25 \mathrm{~cm} \mathrm{H}_{2} \mathrm{O}\right) ; \mathrm{SpO} 2<95 \%$; and reflux. When hiccup, laryngospasm, bronchospasm, leakage or high inspiratory pressure occurred, we increased the depth of anesthesia or administered neuromuscular blockers. If high inspiratory pressure or audible leakage was detected, we adjusted the position of the LMA or the patient's head.

After one hour in the recovery room, a structured interview was conducted to investigate the patient's sore throat, which was recorded by ablinded observer. The severity of sore throat was graded according to three grades: mild, moderate, or severe. Three hours after the operation, a blinded observer was admitted to the ward to follow up complications related to LMA placement (postoperative oral bleeding, maxillary hematoma, and vocal cord paralysis).

\section{Statistical analysis}

The required sample size to show non-inferiority of Jcerity Endoscoper Airway versus LMA Supreme based on the primary outcome measure of one-time implantation rate was estimated to be 176 patients. This estimation was calculated on the basis of an expected one-time implantation rate of $93 \%$ for Jcerity Endoscoper Airway and $\mathbf{9 6 . 7 \%}$ for LMA Supreme[13] with non-inferiority established if the upper limit of the two-sided $95 \% \mathrm{Cl} \%$ of the absolute risk difference was less than $12 \%$ (non-inferiority margin) and the sample size was set to ensure at least $80 \%$ power $(1-\beta=0.8)$ at a significance level of $a=5 \%$. Statistical analysis was performed using Statistical Package for Social Sciences (SPSS Inc., version 24.0 for Windows, Chicago, IL, USA). Continuous variables were evaluated for normality using the KolmogorovSmirnov statistic ( $P>0.05$ indicated normality). Normally distributed continuous variables were presented as mean \pm standard deviation (SD). An unpaired t-test was used to compare continuous variables, the Mann-Whitney test was used to compare skewed variables and the $\chi 2$ test or Fischer exact test was used 
to compare categorical variables between Jcerity Endoscoper and LMA Supreme groups. All statistical tests were two-sided and $p$ values $<0.05$ were considered significant.

\section{Results}

No differences were observed between the two groups in terms of age, sex, BMI or duration of surgery (Table 1).

Table 1

Patient Characteristics and Surgical Data

\begin{tabular}{|llll|}
\hline Patients & Jcerity Endoscoper $(\mathbf{n}=\mathbf{9 2})$ & LMA Supreme $(\mathbf{n = 9 0})$ & $\boldsymbol{P}$ \\
\hline Age $(\mathrm{y})$ & $57.78 \pm 1.24$ & $55.78 \pm 1.29$ & 0.75 \\
\hline Sex $(\mathrm{F} / \mathrm{M})$ & $30 / 62$ & $31 / 59$ & 0.88 \\
\hline BMI & $23.91 \pm 0.32$ & $24.33 \pm 0.29$ & 0.26 \\
\hline Duration of surgery $(\mathrm{min})$ & $105.50 \pm 3.42$ & $101.70 \pm 3.70$ & 0.52 \\
\hline Data are expressed as number of patients or mean \pm SD or absolute numbers, BMI : body mass index. \\
\hline
\end{tabular}

All patients successfully completed the operation and returned to the ward safely. No adverse events of ventilation occurred during the operation. Except for one patient in LMA Supreme group who needed to be changed to endotracheal intubation, all patients successfully underwent LMA. The success rate of onetime implantation in the Jcerity Endoscoper Airway group was $93.48 \%$, and that of the LMA Supreme group was $96.67 \%$. There was no significant difference between the two groups. There were five patients in the Jcerity Endoscoper Airway group and two in the LMA Supreme group who required a second attempt to implant by evacuating the gas in the cuff or lifting the mandible. One patient in the Jcerity Endoscoper group was successfully ventilated by changing the LMA to one size smaller, and there was no significant difference between the two groups. No differences were observed between the two groups in terms of LMA insertion times, number of insertion attempts, quality of ventilation, peak airway pressure, or FBS view of VC (vocal cords) (Table 2). 
Table 2

Airway Management Details

\begin{tabular}{|llll|}
\hline Management Details & Jcerity Endoscoper $(n=92$ & $\begin{array}{l}\text { LMA Supreme }(n= \\
90)\end{array}$ & $P$ \\
\hline LMA insertion & & & \\
\hline Time (s): & $20.64 \pm 0.40$ & $20.62 \pm 0.35$ & 0.25 \\
\hline No. attempts(1/2/3): & $86 / 5 / 1$ & $87 / 2 / 1$ & 0.53 \\
\hline one-time implantation rate: & $93.48 \%$ & $96.67 \%$ & 0.50 \\
\hline Quality of ventilation $(1 / 2 / 3)$ & $89 / 3 / 0$ & $88 / 2 / 1$ & 0.55 \\
\hline Peak airway pressure $\left(\mathrm{cm} \mathrm{H}_{2} \mathrm{O}\right)$ & $16.87 \pm 0.27$ & $17.02 \pm 0.25$ & 0.68 \\
\hline $\begin{array}{l}\text { FBS view of VC } \\
\text { (optimal/suboptimal) }\end{array}$ & $87 / 5$ & $86 / 4$ & 0.76 \\
\hline $\begin{array}{l}\text { Data are expressed as number of patients or mean } \pm \text { SD. FBS: fiberscope; LMA: laryngeal mask } \\
\text { airway; VC: vocal cords. }{ }^{*} P \leq 0.05 \text { (Jcerity Endoscoper Airway vs.LMA Supreme). }\end{array}$ & \\
\hline
\end{tabular}

Compared with LMA Supreme groups, the sealing pressures of the airway at T1-T4 in the Jcerity Endoscoper Airway group were $27.82 \pm 4.15,27.34 \pm 3.89,26.25 \pm 3.02,25.12 \pm 3.01$, respectively. There was no significant difference between the two groups $(P>0.05)$ (Fig. 3$)$.

In patients receiving perioperative anticoagulation, operations on the airway can easily cause various injuries. In the LMA Supreme group, there were five patients with oral bleeding after returning to the ward, and one patient was estimated to bleed more than $200 \mathrm{ml}$. Based on visual laryngoscopy, the causes of bleeding were as follows: two were oral mucosal injuries, two were maxillary hematomas, and one was a lingual frenulum injury. In contrast, there was no significant oral bleeding in the Jcerity Endoscoper Airway group. Only one patient felt a sore throat, and visual laryngoscopy after surgery revealed a tongue frenulum injury. Compared with the LMA Supreme group, the degrees of mask body blood staining and sore throat were significantly lower in the Jcerity Endoscoper Airwaygroup $(P=0.04$ and $P=0.03$ ). Complications such as laryngeal nerve injury, vocal cord paralysis, and arytenoid cartilage dislocation were not observed in either group(Table 3). 
Table 3

Postoperative complications related to laryngeal mask placement

\begin{tabular}{|llll|}
\hline Associated complications & $\begin{array}{l}\text { Jcerity Endoscoper } \\
(\mathbf{n}=93)\end{array}$ & $\begin{array}{l}\text { LMA Supreme }(\mathbf{n} \\
\mathbf{9} 90)\end{array}$ & $P$ \\
\hline $\begin{array}{l}\text { Blood staining degree of the } \\
\text { mask(mild/moderate/sever) }\end{array}$ & $3 / 1 / 0$ & $12 / 3 / 1$ & $0.04^{*}$ \\
\hline sore throat (mild/moderate/sever) & $6 / 1 / 0$ & $17 / 3 / 1$ & $0.03^{*}$ \\
\hline Postoperative oral bleeding & 0 & 5 & $0.02^{*}$ \\
\hline Tongue frenulum injury & 1 & 2 & 0.55 \\
\hline Laryngeal nerve injury or vocal cord paralysis & 0 & 0 & \\
\hline Arytenoid cartilage dislocation & 0 & 0 & 0.15 \\
\hline Maxillary hematoma & 0 & 2 & \\
\hline Difficulty swallowing & 3 & 7 & \\
\hline Lingual nerve palsy & 0 & 0 & \\
\hline Data are represented as number of patients or mean \pm SD. & & \\
\hline$* P \leq 0.05$ (Jcerity Endoscoper Airway vs.LMA Supreme). & & \\
\hline
\end{tabular}

\section{Discussion}

The global prevalence of adult cerebral aneurysms is estimated to be between $0.65 \%$ and $3.2 \%$. With the continuous improvement of screening and imaging technology, the detection rate of cerebral aneurysms has continued to increase[14]. For the treatment of cerebral aneurysms, craniotomy clipping presents greater surgical trauma and risk, while endovascular interventional therapy achieves occlusion of intracranial aneurysms by implanting stents into the aneurysm and releasing a coil. Due to its minimally invasive nature, safety, and effectiveness, it has been widely used in clinical practice in recent years [4, 15-19]. Cerebral aneurysm embolization is an intravascular operation associated with mild stimulation. The operation time is usually 1-2 hours. During the operation, the patient's head should be kept totally still. During general anesthesia, it is necessary to perform mechanical ventilationin order not to interfere with the contrast imaging.

The primary goal of anesthesia management for cerebral aneurysm embolization are to maintain the stability of perioperative circulation, to keep the patient's head immobilization during the operation and to promptly deal with intraoperative complications [17]. At present, the most common anesthesia methods are sedation and general anesthesia. Simple sedation allows convenient and frequent neurological evaluations, avoiding tracheal intubation and related hemodynamic changes. The disadvantage is that it does not protect the airway, and there is a risk of reflux aspiration and potential hypoxemia and hypercapnia. Sudden movements and delayed management of neurological emergencies may also occur 
$[20,21]$. The main advantages of general anesthesia are that patients remain still, improving imaging quality, and increasing safety and efficiency. If complications occur, it is convenient to deal with them. However, hemodynamic fluctuations caused by anesthesia induction and tracheal intubation and the inability to continuously assess the patient's neurological status are its main disadvantages [14]. The traditional method of general anesthesia for cerebral aneurysm embolization is to use tracheal intubation and general anesthesia. General anesthesia with tracheal intubation requires deeper anesthesia and proper muscle relaxation during the induction as well as maintenance of anesthesia. During tracheal intubation and extubation, there are often substantial hemodynamic fluctuations that are extremely harmful to patients with aneurysms; these often cause serious adverse reactions such as cerebral aneurysm rupture and hemorrhage [22, 23].

As a supraglottic ventilation tool, LMA avoids direct irritation to glottis and trachea. It has been widely used since its introduction in the US in 1988[12, 24, 25]. The double lumen LMA is used in various operations because it greatly reduces reflux and aspiration [26]. A study showed that general anesthesia using laryngeal mask airway not only reduced hemodynamic fluctuation during surgery, but it also allowed the patients to be awakened quickly [27]. Nevertheless, although LMA placement avoids direct irritation and injury to the glottis and trachea, it can damage soft tissues of the mouth, pharynx and larynx. Pharyngeal pain and bleeding are common postoperative complications [28]. During cerebral aneurysm embolization with LMA Supreme general anesthesia, we found that patients who received anticoagulant and antiplatelet aggregation therapy experienced significantly increased pharyngeal pain and bleeding after surgery. Therefore, based on the characteristics of this kind of surgery for perioperative anticoagulant patients, we need a ventilation device that not only ensures effective ventilation but also reduces oropharyngeal injuries. As a special type of laryngeal mask, the endoscopic laryngeal mask airway has been shown to be safe and effective in upper gastrointestinal endoscopic surgery [29].There are also reports on its application in minimally invasive cardiovascular surgery such as atrial fibrillation radiofrequency ablation[30].

Based on its special material and structural characteristics of the Jcerity Endoscoper Airway, we used it to manage the airway in cerebral aneurysm embolization. On one hand, the large-volume inflatable cuff is made of silica gel material, which has high elasticity and flexibility, on the other hand, it fits the anatomical curve of each patient's oropharynx, and forms an effective sealing area in the throat, in particular, it could avoid injury to the throat caused by excessive inflation compression. Studies have shown that the incidence of postoperative sore throat is not only related to implantation trauma, but also to long-term compression of the laryngeal mask [31].

As a new type of LMA specially developed for upper gastrointestinal endoscopy, the Jcerity Endoscoper Airway's innovation is to add a dedicated endoscopic channel (20*22 mm inner diameter) that runs in parallel with an independent airway channel with a terminal cuff. Its endoscopic examination channel is not completely closed, which greatly reduces the friction area between the laryngeal mask and the posterior pharynx wall during the insertion process. The most important thing is that the main tube adopts a fixed angle and streamlined design, and the head end of the cuff is a semi-open buffer sheet 
structure, which minimizes the resistance damage to the oropharynx and the mucosa around the glottis during the placement process. The LMA Supreme ventilation cavity is reinforced with steel wire. To prevent LMA Supreme folding during the insertion process, a hard guidewire is built in for shaping, and the tip of the suction cavity is relatively hard. Therefore, during insertion, especially when pulling out the guidewire, the head end of the LMA Supreme inflicts substantial resistance damage to the throat. In our study, we found that both the LMA Supreme and the Jcerity Endoscoper Airway provided safe and effective airway protection for patients undergoing general anesthesia for cerebral aneurysm embolization. There were no significant differences in terms of success rate of implantation, implantation time, difficulty of implantation, fiberoptic bronchoscopy field of vision, ventilation quality, intraoperative airway pressure, orsealing pressure. Due to the specificity of cerebral aneurysm embolization, compared with the LMA Supreme group, the blood staining degree of the mask, postoperative sore throat, postoperative oral hemorrhage in the Jcerity Endoscoper Airway group were significantly lower, which may be related to its particular material composition and structure.

In conclusion, compared with LMA Supreme, Jcerity Endoscoper Airway significantly reduced postoperative pharyngeal pain and oral bleeding in patients undergoing interventional embolization under general anesthesia for treatment of cerebral aneurysms.

\section{Declarations}

The authors declare that we have no conflicts of interest to this work. We declare that we do not have any commercial or associative interest that represents a conflict of interest in connection with the work submitted.

\section{Author Contributions}

Junfei Zhou and Lu Li contributed to study design and data collection and analysis, and also contributed significantly to manuscript preparation and they contributed equally to this study;

Fang Wang performed the experiment and helped with the patient recruitment;

Yunqi Lv writing up of the first draft of the paper and helped perform the analysis with constructive discussions.

\section{References}

1. Diaz O, Rangel-Castilla L. Endovascular treatment of intracranial aneurysms. Handb Clin Neurol. 2016;136:1303-9.

2. Kamo T, Akioka N, Kashiwazaki D, Koh M, Kuwayama N, Kuroda S. [Clinical Features and Findings in Patients with Unruptured Intracranial Aneurysms Aged $\geq 80$ Years]. No Shinkei Geka. 2017;45:15-9.

3. Desai B, Soldozy S, Desai H, Kumar J, Shah S, Raper DM, Park MS: Evaluating the safety and efficacy of various endovascular approaches for the treatment of infectious intracranial aneurysms: A 
systematic review. World Neurosurg 2020.

4. Pierot L, Barbe C, Nguyen HA, Herbreteau D, Gauvrit JY, Januel AC, Bala F, Comby PO, Desal H, Velasco S, Aggour M, Chabert E, Sedat J, Trystram D, Marnat G, Gallas S, Rodesch G, Clarençon F, Soize S, Gawlitza M, Spelle L, White P. Intraoperative Complications of Endovascular Treatment of Intracranial Aneurysms with Coiling or Balloon-assisted Coiling in a Prospective Multicenter Cohort of 1088 Participants: Analysis of Recanalization after Endovascular Treatment of Intracranial Aneurysm (ARETA) Study. Radiology. 2020;296:E130-0E133.

5. Rizvi A, Seyedsaadat SM, Alzuabi M, Murad MH, Kadirvel R, Brinjikji W, Kallmes DF. Long-Term Rupture Risk in Patients with Unruptured Intracranial Aneurysms Treated with Endovascular Therapy: A Systematic Review and Meta-Analysis. AJNR Am J Neuroradiol. 2020;41:1043-8.

6. Sirakov A, Bhogal P, Möhlenbruch M, Sirakov S. Endovascular treatment of patients with intracranial aneurysms: feasibility and successful employment of a new low profile visible intraluminal support (LVIS) EVO stent. Neuroradiol J 2020:1971400920941402.

7. Lee CZ, Gelb AW. Anesthesia management for endovascular treatment. Curr Opin Anaesthesiol. 2014;27:484-8.

8. Kang E, Lee KH, Park JH. Comparison of Two Methods of Anesthesia Using Patient State Index: Propofol Versus Sevoflurane During Interventional Neuroradiology Procedure. Anesth Pain Med. 2019;9:e87518.

9. Griessenauer CJ, Shallwani H, Adeeb N, Gupta R, Rangel-Castilla L, Siddiqui AH, Levy El, Boone MD, Thomas AJ, Ogilvy CS. Conscious Sedation Versus General Anesthesia for the Treatment of Cerebral Aneurysms with Flow Diversion: A Matched Cohort Study. World Neurosurg. 2017;102:1-5.

10. Naggara ON, Lecler A, Oppenheim C, Meder JF, Raymond J. Endovascular treatment of intracranial unruptured aneurysms: a systematic review of the literature on safety with emphasis on subgroup analyses. Radiology. 2012;263:828-35.

11. Norman A, Nohra C, Starke RM, Mario Z, Rodney B: Unruptured Cerebral Aneurysms: Evaluation and Management. The Scientific World Journal,2015,(2015-6-4) 2015, 2015:1-10.

12. Yu SH, Beirne OR. Laryngeal mask airways have a lower risk of airway complications compared with endotracheal intubation: a systematic review. Journal of Oral Maxillofacial Surgery Official Journal of the American Association of Oral Maxillofacial Surgeons. 2010;68:2359-76.

13. López AM, Valero R, Hurtado P, Gambús P, Pons M, Anglada T. Comparison of the LMA Supreme ${ }^{T M}$ with the LMA Proseal ${ }^{\mathrm{TM}}$ for airway management in patients anaesthetized in prone position. $\mathrm{Br} \mathrm{J}$ Anaesth. 2011;107:265-71.

14. De Sloovere VT. Anesthesia for embolization of cerebral aneurysms. Curr Opin Anaesthesiol. 2014;27:431-6.

15. Molyneux AJ, Kerr RS, Yu LM, Clarke M, Sneade M, Yarnold JA, Sandercock P, International Subarachnoid Aneurysm Trial (ISAT) Collaborative Group. International subarachnoid aneurysm trial (ISAT) of neurosurgical clipping versus endovascular coiling in 2143 patients with ruptured 
intracranial aneurysms: a randomised comparison of effects on survival, dependency, seizures, rebleeding, subgroups, and aneurysm occlusion. Lancet. 2005;366:809-17.

16. Cowan JA, Ziewacz J, Dimick JB, Upchurch GR, Thompson BG. Use of endovascular coil embolization and surgical clip occlusion for cerebral artery aneurysms. J Neurosurg. 2007;107:5305.

17. Ishibashi T, Murayama Y, Ikeuchi S, Saguchi T, Abe T: Unruptured intracranial aneurysms: Natural history, risk of rupture, and clinical outcome of endovascular treatment as a first line of therapy. 2007.

18. Luther E, McCarthy DJ, Brunet MC, Sur S, Chen SH, Sheinberg D, Hasan D, Jabbour P, Yavagal DR, Peterson EC, Starke RM. Treatment and diagnosis of cerebral aneurysms in the post-International Subarachnoid Aneurysm Trial (ISAT) era: trends and outcomes. J Neurointerv Surg. 2020;12:682-7.

19. Mokin M, Primiani CT, Ren Z, Piper K, Fiorella DJ, Rai AT, Orlov K, Kislitsin D, Gorbatykh A, Mocco J, De Leacy R, Lee J, Vargas Machaj J, Turner R, Chaudry I, Turk AS. Stent-assisted coiling of cerebral aneurysms: multi-center analysis of radiographic and clinical outcomes in 659 patients. $\mathrm{J}$ Neurointerv Surg. 2020;12:289-97.

20. Park SD, Kim JH, Chang CH, Jung YJ. Procedure-related Complication Rate for the Endovascular Treatment of Aneurysmal Subarachnoid Hemorrhage under Local Anesthesia. J Cerebrovasc Endovasc Neurosurg. 2016;18:215-22.

21. Ihn YK, Shin SH, Baik SK, Choi IS. Complications of endovascular treatment for intracranial aneurysms: Management and prevention. Interv Neuroradiol. 2018;24:237-45.

22. Ozhan MO, Eskin MB, Atik B, Suzer MA, Capalar CO. Laryngeal mask airway for general anesthesia in interventional neuroradiology procedures. Saudi Med J. 2019;40:463-8.

23. Zhang Q, Sun Y, Wang B, Wang S, Mu F, Zhang Y. Comparative study of the Ambu $\AA^{\circledR}$ AuraOnce ${ }^{\mathrm{TM}}$ laryngeal mask and endotracheal intubation in anesthesia airway management during neurosurgery. J Int Med Res. 2020;48:300060520902606.

24. Xu R, Lian Y, Li WX. Airway Complications during and after General Anesthesia: A Comparison, Systematic Review and Meta-Analysis of Using Flexible Laryngeal Mask Airways and Endotracheal Tubes. PLoS One. 2016;11:e0158137.

25. Osborn IP, Cohen J, Soper RJ, Roth LA. Laryngeal mask airway-a novel method of airway protection during ERCP: Comparison with endotracheal intubation. Gastrointest Endosc. 2002;56:122-8.

26. Qamarul Hoda M, Samad K, Ullah H. ProSeal versus Classic laryngeal mask airway (LMA) for positive pressure ventilation in adults undergoing elective surgery. Cochrane Database Syst Rev. 2017;7:CD009026.

27. Wiebers DO, Whisnant JP, Huston J 3rd, Meissner I, Brown RD Jr, Piepgras DG, Forbes GS, Thielen K, Nichols D, O'Fallon WM, Peacock J, Jaeger L, Kassell NF, Kongable-Beckman GL, Torner JC. International Study of Unruptured Intracranial Aneurysms Investigators: Unruptured intracranial aneurysms: natural history, clinical outcome, and risks of surgical and endovascular treatment. Lancet. 2003;362:103-10. 
28. van Esch BF, Stegeman I, Smit AL. Comparison of laryngeal mask airway vs tracheal intubation: a systematic review on airway complications. J Clin Anesth. 2017;36:142-50.

29. Terblanche NCS, Middleton C, Choi-Lundberg DL, Skinner M. Efficacy of a new dual channel laryngeal mask airway, the LMAGastro Airway, for upper gastrointestinal endoscopy: a prospective observational study. Br J Anaesth. 2018;120:353.

30. Saxena S, Aminian A, Nahrwold DA, Daper A. LMA Gastro Airway Seen Through the Eyes of a Cardiac Anesthesiologist. J Cardiothorac Vasc Anesth. 2019;33:2365-6.

31. Grady DM, McHardy F, Wong J, Jin F, Tong D, Chung F. Pharyngolaryngeal morbidity with the laryngeal mask airway in spontaneously breathing patients: does size matter. Anesthesiology. 2001;94:760-6.

\section{Figures}

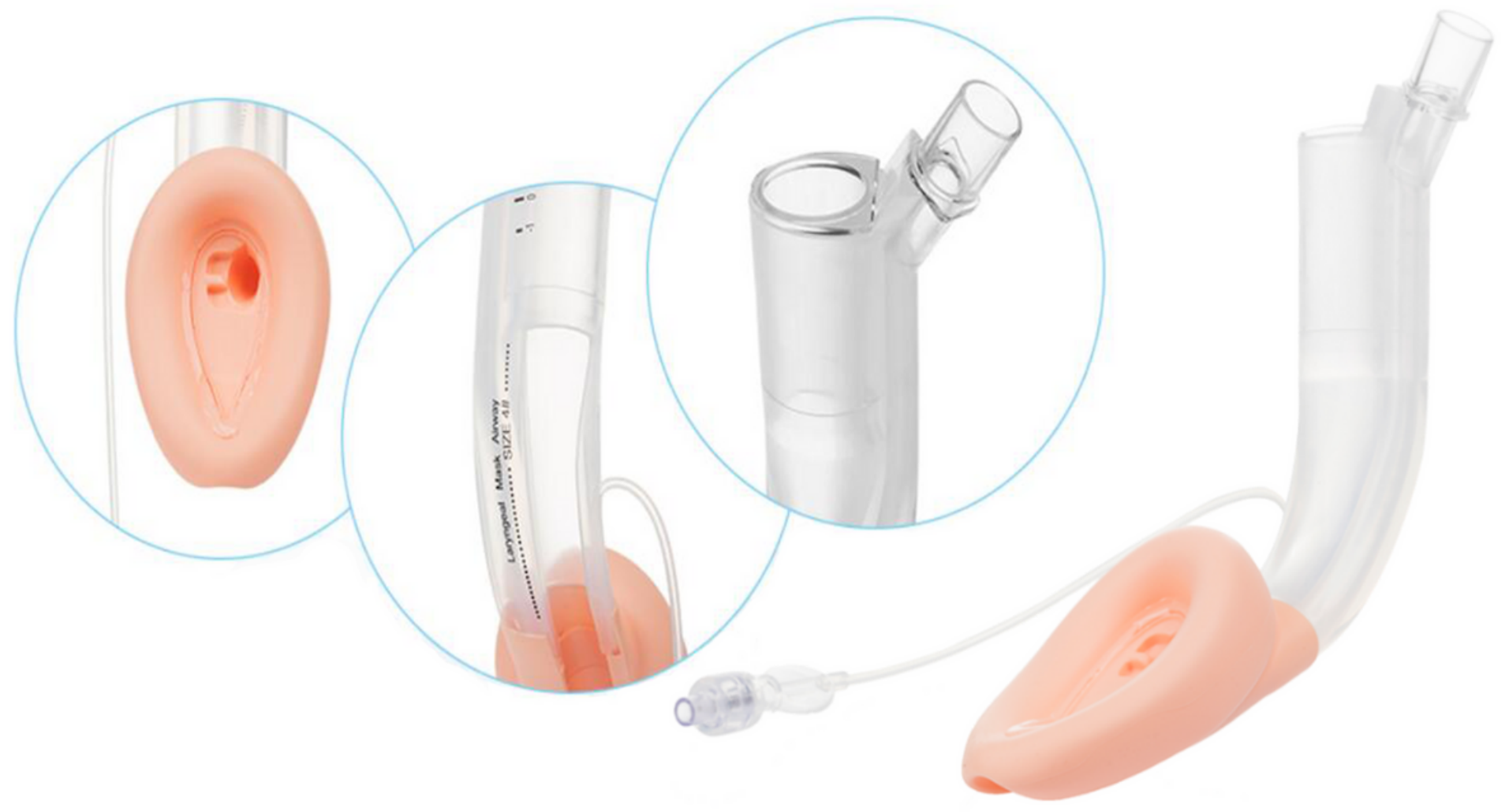

\section{Figure 1}

The design features of Jcerity Endoscoper Airway. 


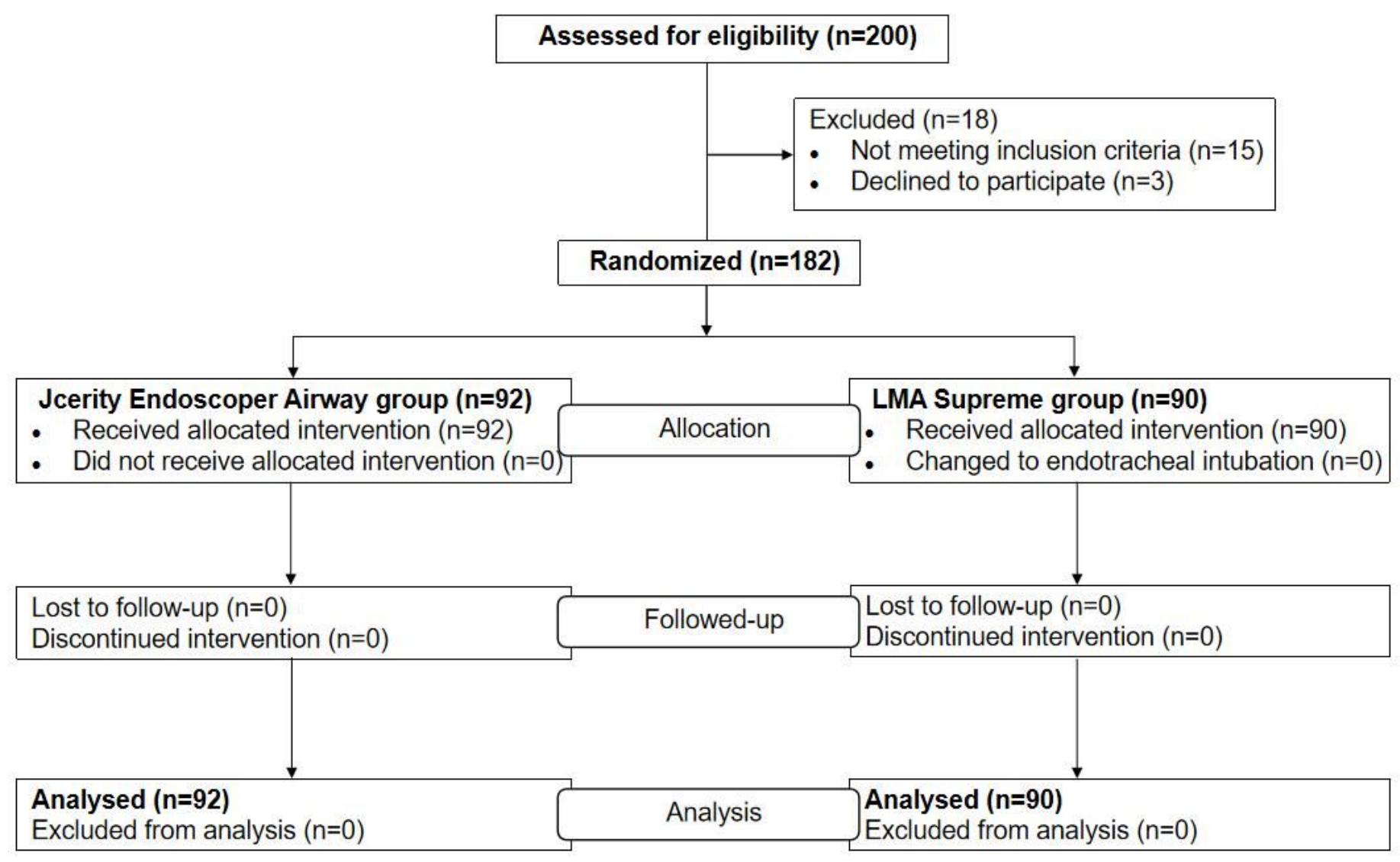

\section{Figure 2}

Patient enrolment and flow. 


\section{Jcerity Endoscoper \\ שas LMA Supreme}

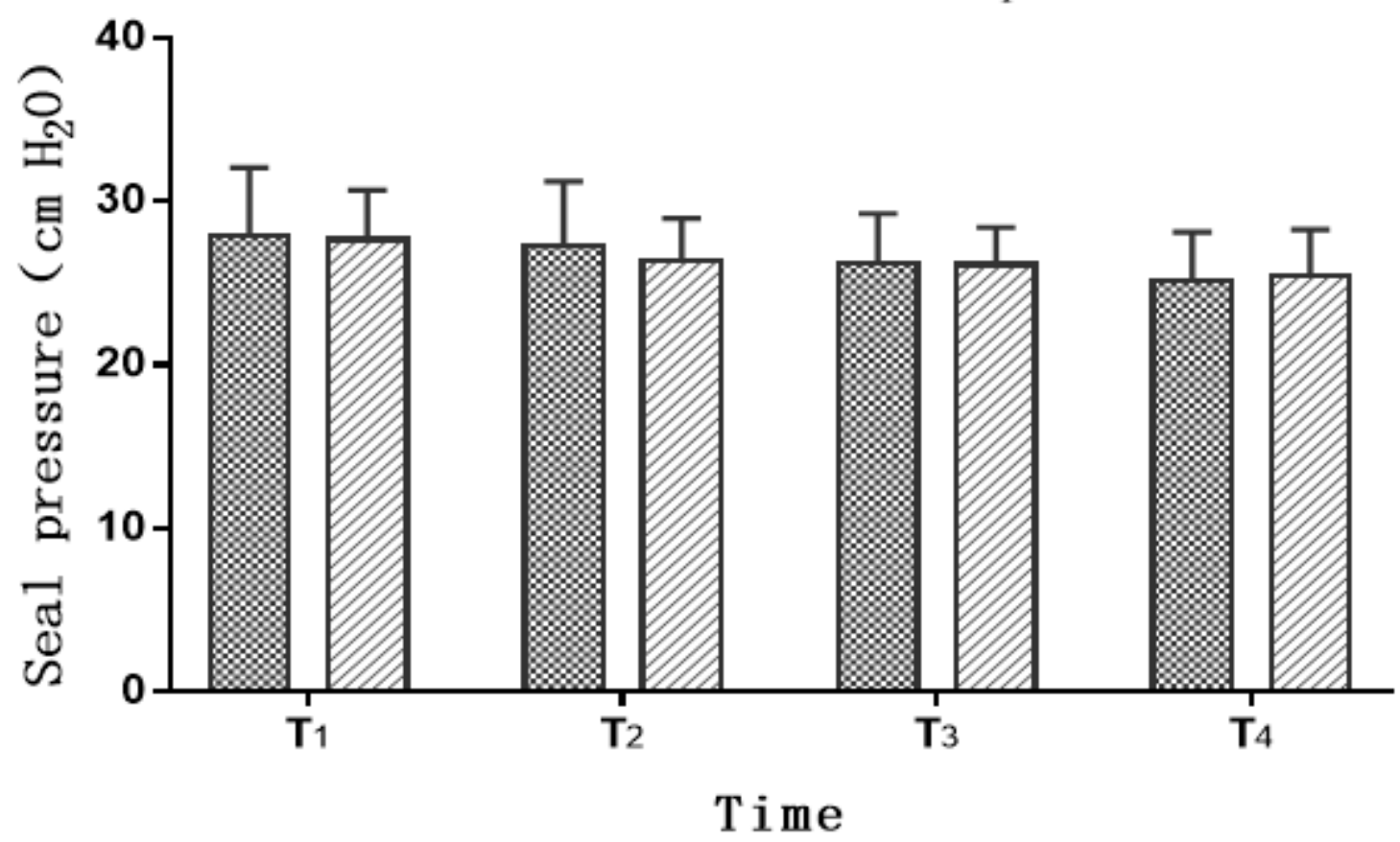

Figure 3

Seal pressure of Jcerity Endoscoper Airway and LMA Supreme groups at various points. Data are represented as number of patients or mean \pm SD. There was no difference between the two groups. *P $\leq 0.05$ (Jcerity Endoscoper Airway vs. LMA Supreme). 